https://dx.doi.org/10.4314/jpb.v17i2.7

Vol. 17 no. 2, pp. 131-141 (September 2020)

http://ajol.info/index.php/jpb

\section{Journal of \\ PHARMACY AND BIORESOURCES}

\title{
Prevalence and antibiotic susceptibility of community acquired methicillin resistant Staphylococcus aureus from healthy students of University of Jos
}

\author{
Patrick O. OLORUNFEMI $^{1 *}$, Josiah A. ONAOLAPO ${ }^{2}$ and Yakubu K.E. IBRAHIM ${ }^{2}$ \\ ${ }^{I}$ Department of Pharmaceutical Microbiology and Biotechnology, University of Jos, Jos. Nigeria. \\ ${ }^{2}$ Department of Pharmaceutical Microbiology, Ahmadu Bello University, Zaria. Nigeria.
}

Received 22 ${ }^{\text {nd }}$ May 2020; Accepted $3^{\text {rd }}$ September 2020

\begin{abstract}
Staphylococcus aureus is a virulent pathogen that is currently, not only the most common cause of nosocomial infections worldwide, but increasingly also, a community associated pathogen. This study aimed at determining the prevalence and susceptibility of methicillin resistant $S$. aureus (MRSA) isolated from apparently healthy university student population in Jos Nigeria. Two hundred and seventeen (217) urine samples were screened for Staphylococcus aureus. Isolates were characterized by conventional cultural and biochemical methods including rapid test kits (MicrogenID test kit). Their susceptibility profiles were determined against a panel of eleven antibiotics using the method of the Clinical and Laboratory Standard Institute (CLSI). The MRSA status were confirmed phenotypically using oxacillin and cefoxitin as markers. Of the 217 samples collected, 171 (78.8\%) were positive for Staphylococcal species, while 135 of these were coagulase positive and were classed as S. aureus. Seventy-three (73) of these were identified as $S$. aureus with the MicrogenID test kit. Isolates were resistant to at least one of the antibiotics used in the study. Seventy-one (71) of $73 \mathrm{~S}$. aureus isolates were methicillin resistant. The susceptibility pattern observed in community isolates implies that any outbreak of infections caused by these strains may pose a threat to public health.
\end{abstract}

Keywords: Staphylococcus aureus; Prevalence; Antimicrobial resistance; MRSA, Susceptibility

\section{INTRODUCTION}

Staphylococcus aureus has been known to be a major and versatile pathogen causing a wide spectrum of clinical manifestations, such as skin and soft tissue infections, pneumonia, septicemia, pleuropulmonary, osteomyelitis, endocarditis, arthritis, gastroenteritis, meningitis, urinary tract infections, device-related infections and toxinoses (such as food poisoning, septic shock, scalded skin syndrome and toxic shock syndrome) [1-4] with beta-lactam antibiotics being the drugs of choice for therapy. Since the introduction of methicillin into clinical use in 1961, the occurrence of methicillin-resistant S. aureus (MRSA) has steadily increased and nosocomial infections caused by such isolates have become a serious problem worldwide and the emergence of community acquired MRSA is another growing risk [5,6]. MRSA isolates are resistant to the $\beta$-lactam antibiotics. Vancomycin is used to treat MRSA infections. Considerable research efforts have been put forward to improve our understanding of

*Correspondence. E-mail: patola56@gmail.com Tel: +234-8037013042.

ISSN 0189-8442

(cc) BY-NC 202. Published by Faculty of Pharmaceutical Sciences, University of Jos, Nigeria. Under Creative Commons Attribution-NonCommercial 4.0 International License. https://creativecommons.org/licenses/by-nc/4.0/ 
complex pathogenesis of $S$. aureus. Despite these efforts, the burden of staphylococcal infections is still on the rise. The unresolved concern regarding this pathogen, include the nature of the driving forces behind the rise and decline of methicillin-resistant $S$. aureus (MRSA) clones; the mechanisms by which a commensal becomes a pathogen; the molecular underpinnings of toxin overexpression in hyper virulent MRSA clones such as USA300; and the repeated failures of anti-S. aureus vaccine approaches [7].

Staphylococcus aureus has a wide host range, diverse cellular and environmental lifestyles, and the capability to evolve to highly drug-resistant forms that can cause diseases with epidemic potential [8]. Staphylococcus aureus exhibits two distinct lifestyles - a commensal asymptomatic state where it is harboured by $20-50 \%$ of the general populations at the anterior nares; and the acute state at which it attacks specific tissues and pathogenicity follows consequently. Generally, Staphylococcus aureus causes infections by self- inoculation (a process whereby the organism breaches the natural barrier) or by direct or indirect contact [8]. The spread or containment of an infection depends on a complex interplay between Staphylococcus aureus virulence determinants and the host defense mechanism [9]. S. aureus colonises mainly the nasal passages, but it may be found regularly in most other anatomical locales. It is not typically an invasive pathogen, however, it invokes symptoms by the production of a variety of extracellular proteins (virulence factors), some cell-bound, others secreted, which participate in the initiation and establishment of an infection [10,11].

Antibiotic resistance is a critical threat to public health. Some of the factors facilitating antimicrobial resistance are irrational use of antibiotics by health professionals, unskilled practitioners and the public; the ready availability of antibiotics as over the counter drugs, poor quality of antibiotics, crowding and unhygienic conditions; inadequate hospital infection control practices, excessive use in food producing animals, inadequate surveillance, and limited funds $[12,13]$. The continuous rise of resistance of $S$. aureus against antibiotics presents a challenge with the management of MRSA infections. The prevalence of MRSA is on the rise globally. It is estimated that MRSA kills about 50, 000 patients every year in the United States and Europe alone [13]. Data is not available to determine the number of patients that die from MRSA in Nigeria. However, assessing the studies undertaken in Nigeria and the stated prevalence in various location [14-20], it has become necessary to contain antimicrobial resistance. Based on WHO's antimicrobial resistance global report on surveillance, S. aureus is 1 of 7 bacteria of international concern [21]. The developed countries have formulated stiff policies and stimulated surveillance methods; however, the developing countries need to step up efforts on their approaches to fight antimicrobial resistance [22]. Although Nigeria has developed her National Action Plan for antimicrobial resistance, 2017-2022, its implementation three years later is still at the rudimentary stage as its impact is yet to be felt. In Nigeria, antibiotics are still bought over the counter despite the years of antimicrobial resistance. Thus increasing the selective pressure of antibiotics when its reduction is a strategy for containing antimicrobial resistance. Antibiotic stewardship program under which reduction of selective pressure on antibiotics falls appear not be fully in place in most hospitals in Nigeria. A study by Abubakar and colleague revealed that after years of advocation for antibiotic stewardship only a few tertiary hospitals (training centres for health professionals) have formal antimicrobial stewardship team [23]. Fadare and co-workers also observed significant inadequacies in the availability of antimicrobial stewardship programs in tertiary 
hospitals in Nigeria [24]. The setting up of the national antimicrobial surveillance system is still at infant stage. There is limited collaboration amongst stakeholders and poor public awareness. Meanwhile, MRSA continues to develop resistance to available antibiotics and few antimicrobial agents are in the pipeline as there is limited focus on antibiotic research. Coordinated and collaborative efforts are urgently needed to contain antimicrobial resistance in order to avert a major public health disaster.

This study aims at determining the carriage/prevalence and the antibiotic susceptibility profile of MRSA isolates from apparently healthy students of the University of Jos community in Plateau State, Nigeria. It buttresses the need for the National Action Plan for antimicrobial resistance to be urgently implemented.

\section{EXPERIMENTAL}

Sample collection. Urine samples were collected from healthy volunteers who were not on any antibiotics (or had not taken any antibiotics) three-four weeks before samples were collected. They included male and female science and arts students. Volunteers were given dry, sterile universal bottles and instructed how to collect the mid-stream urine. The women were instructed to clean the area around the urethral opening with water and towel dry before collecting the urine samples. Convenience sampling method was used targeting 200 to 250 volunteers. The samples so collected were taken to the laboratory and analysed within four hours $(4 \mathrm{~h})$. A total number of 217 urine samples were collected.

Informed consent. All the participants in the study gave informed consent following detailed explanation of study and assurance of anonymity.

Preparation of growth media. The media were reconstituted according to the manufacturer's directive and sterilized at $121^{\circ} \mathrm{C}$ for 15 minutes. The media were freshly prepared as required. For physiological saline, $9 \mathrm{~g}$ of sodium chloride was weighed and dissolved in $1 \mathrm{~L}$ of distilled water and sterilized in an autoclave at $121^{\circ} \mathrm{C}$ for 15 minutes. This was used in diluting the bacterial culture as required.

Isolation of organism. The urine samples were inoculated into nutrient broth and incubated for $18-24$ hours at $37^{\circ} \mathrm{C}$. The overnight cultures were then streaked on to dried mannitol salt agar plates and incubated at $37^{\circ} \mathrm{C}$ for $24-48$ hours. Characteristic colonies were re-streaked on mannitol salt agar and incubated. Isolated characteristic colonies were noted and inoculated into nutrient broth and nutrient agar slants, incubated at $37^{\circ} \mathrm{C}$ overnight and kept for further characterization to establish their identities.

\section{Characterisation and identification of $S$. aureus isolates.}

Staining. Smears of characteristic colonies were made on a clean slide, fixed, gramstained and viewed under the microscope. Isolates that appeared gram-positive in characteristic clusters were subcultured in duplicates unto Nutrient agar slants and incubated overnight at $37^{\circ} \mathrm{C}$.

Catalase test. The test for the presence of the enzyme catalase was demonstrated by the addition of about $1 \mathrm{~mL}$ of $3 \%$ hydrogen peroxide on a 24-hour slant culture of the isolates. Positive cultures were characterized by rapid evolution of gas bubbles. The test differentiates Staphylococci from Streptococci species.

Coagulase test. The latex test method, employing the BactiStaph latex test kit, was used to differentiate Staphylococcus aureus (coagulase positive) from the coagulase negative Staphylococcus spp. Following the manufacturer's instruction, a drop of the BactiStaph latex reagent was dropped in a circle on the slide provided and with the 
stirring stick, two-three colonies were picked and applied to a dry portion of the circle on the slide and slowly blended with the latex reagent and spread over the entire circle. The slide was rotated in a circular motion for not more than 60 seconds and observed for clumping/agglutination. The same procedure was followed for the positive and negative controls on the same slide but different circles. Large black clumps of agglutination with subsequent loss of black background are positive for coagulase.

Identification using rapid test kits. Microgen TM STAPH-ID kit was used for the final identification of the isolates. Single colonies of 24-hour cultures of the 135 coagulase positive isolates were emulsified in the suspending medium of the Microgen test kit and mixed thoroughly. There were 12 wells and with the aid of sterile Pasteur pipette, 3 drops of the bacterial suspension were added to each well of the strips (Figure 1). Wells 10 and 11 were overlaid with 3 drops of mineral oil. The top of the microwell test strips was sealed with the adhesive tape and incubated at $37^{\circ} \mathrm{C}$. The test strips were read after 24 hours against a template. The readings were fed into a software (MicrogenID software) and the isolates were identified to sub-specie level.

\section{Tests for the susceptibility of $S$. aureus isolates to the antibiotics}

Agar diffusion test. The antibiotic susceptibility patterns of the 171 staphylococcal isolates were determined using the disc diffusion test method of the Clinical Laboratory Standards Institute formerly (NCCLS) [25]. Sterile $20 \mathrm{ml}$ aliquots of Mueller-Hinton agar were poured into sterile Petri dishes and allowed to set. Overnight cultures of the isolates were diluted in normal saline to $0.5 \mathrm{McFarland}$ turbidity standard (ca. $10^{8} \mathrm{cfu} / \mathrm{mL}$ ) and used to flood the MuellerHinton agar plates. The excess inoculum was poured out and the plates dried in the incubator for 10 minutes. The antibiotic discs were applied ensuring that every part of the discs touched the agar. The plates were left at room temperature for one hour before incubating in an inverted position at $37^{\circ} \mathrm{C}$ for $18-24$ hours. The inhibition zone diameters were measured to the nearest millimetre using a transparent plastic rule and classified accordingly as sensitive, intermediate or resistant based on the CLSI interpretive chart for zone sizes [25]. Three determinations were done for each isolate and the average of the readings calculated.

Detection of MRSA. Agar plates containing 6 $\mu \mathrm{g} / \mathrm{mL}$ of oxacillin were prepared and standardized overnight culture of the isolates that were methicillin resistant by agar diffusion test were streaked on the plates and incubated overnight for 24 hours at $37^{\circ} \mathrm{C}$. Growth on the plates were noted. The same procedure was followed for vancomycin using a concentration of $2 \mu \mathrm{g} / \mathrm{mL}$.

\section{RESULTS}

Isolation and characterization. Of the total of 217 samples collected, 171 were positive for Staphylococcus by the cultural method giving a staphylococcal prevalence of $78.8 \%$ (Figure 2). All the 171 isolates grew on Mannitol Salt Agar producing yellow colonies with yellow zones in 24-48 hours and showed characteristic Gram-positive cocci in clusters when Gram stained. Out of the 171 isolates, $49.1 \%$ were catalase positive, $78.9 \%$ were coagulase positive and $57.3 \%$ were B-lactamase producers (Figure 2). Of the culturally identified Staphylococcus isolates, 135 were tested with the Microgen TM STAPH-ID kit and $73(54.1 \%)$ of these were confirmed S. aureus.

Susceptibility of isolates to test antibiotics. The results of the susceptibility of the 171 staphylococcal isolates to the various antibiotics are as shown in Table 1. The isolates demonstrated varying degrees of susceptibility to all the antibiotics. Only $8.2 \%$ (14) and $19.3 \%$ (33) were susceptible to 
penicillin and cefotaxime respectively. Of the quinolones, ciprofloxacin was active against most of the isolates - $88.3 \%$ (151). Interestingly, majority of the isolates $-79.5 \%$ (136) were resistant to ofloxacin which is intriguing as ofloxacin has same mechanism of action as ciprofloxacin. Susceptibility to cotrimoxazole was $63.2 \%$ (108) while vancomycin seems to demonstrate good activity. Cefoxitin and oxacillin had $21.1 \%$ (36) and $39.2 \%$ (67) susceptibility, respectively. The test for detection of MRSA with oxacillin showed that $97.3 \%$ (71 of 73) were methicillin resistant.

Figure 3 depicts the multi-resistant pattern of the isolates to the antibiotics indicating the number of antibiotics to which a certain number of isolates were resistant. Most isolates were resistant to at least one antibiotic while minimum of 3 isolates were resistant to the 11 antibiotics. The number and type of antibiotics to which an isolate was resistant differed in some instances from other isolates. The calculated multi-antibiotic resistant index is shown in Table 2.

\section{DISCUSSION}

Nasal carriage of Staphylococci is the predominant mode of carriage in about 20$80 \%$ of the population [26,27]. However, in this study, of the 217 urine samples taken, Staphylococci isolates were recovered from 171 (78.8\%). Since only 73 were identified to be Staphylococcus aureus and 71 as MRSA, their prevalence in the sample population is $33.6 \%$ and $32.7 \%$ respectively. The conventional cultural method of identifying Staphylococcus aureus gave a higher figure of $135(78.9 \%)$ out of 171 isolates (Fig. 2).

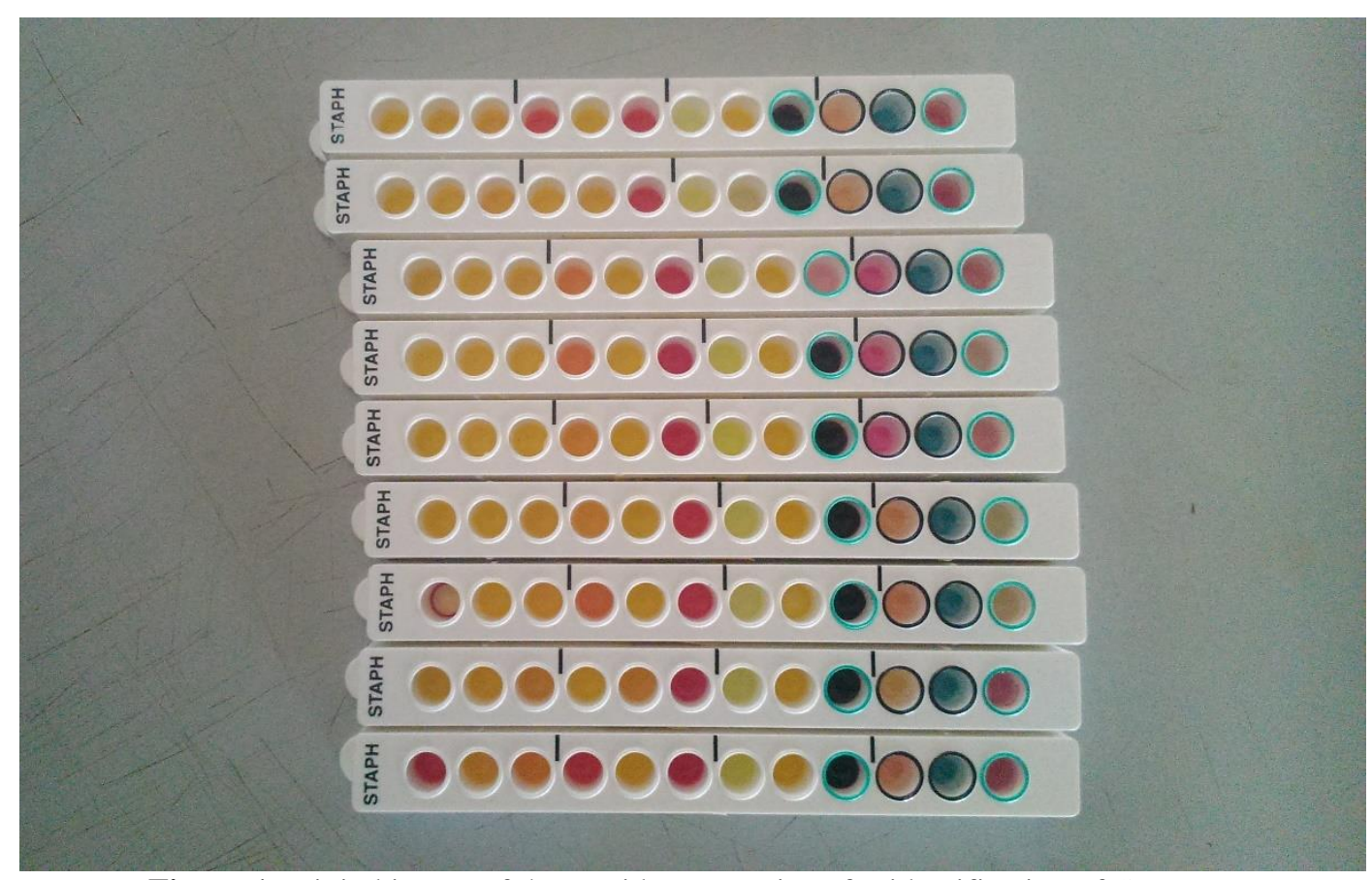

Figure 1: Digital image of the Rapid test reactions for identification of S. aureus 


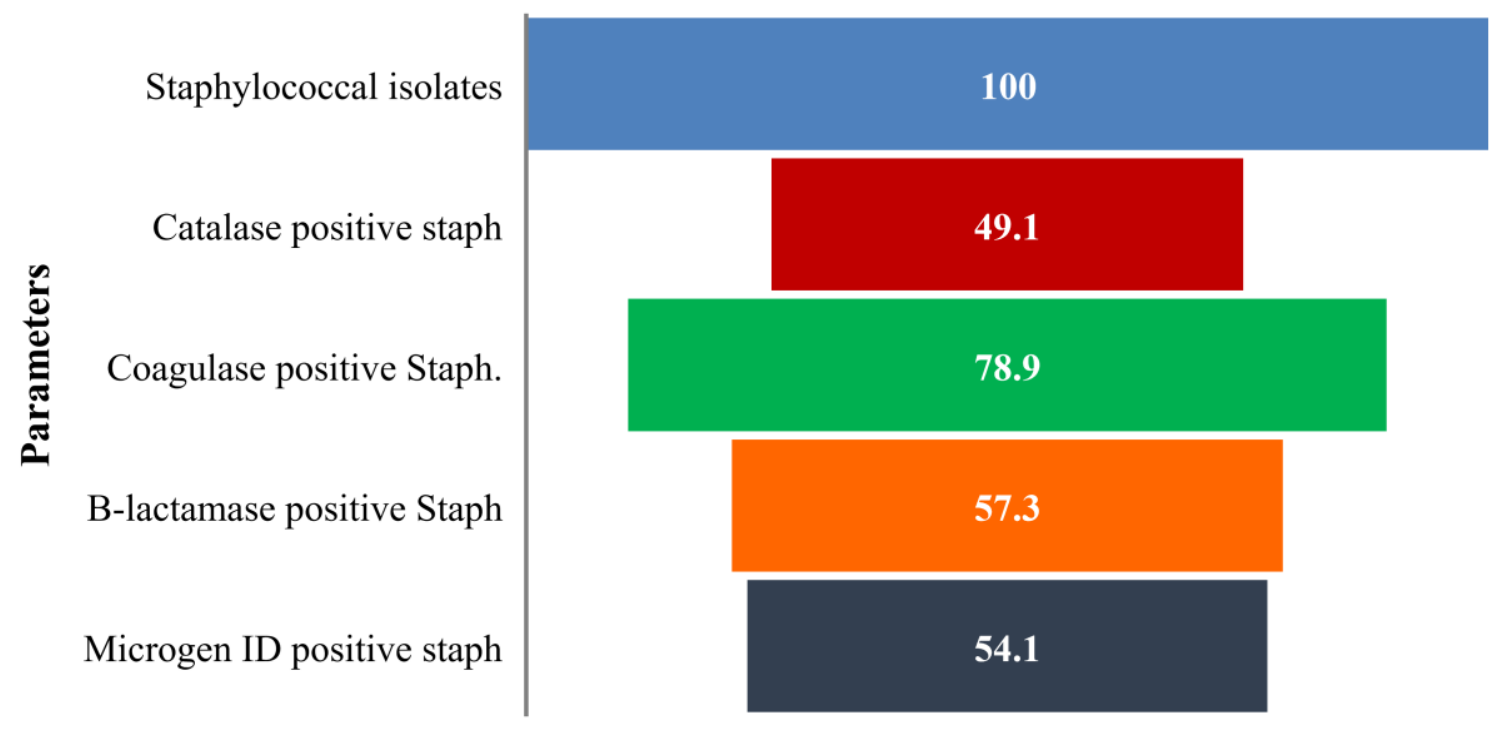

Figure 2: Biochemical characteristics of staphylococcal isolates $(\mathrm{n}=171)$

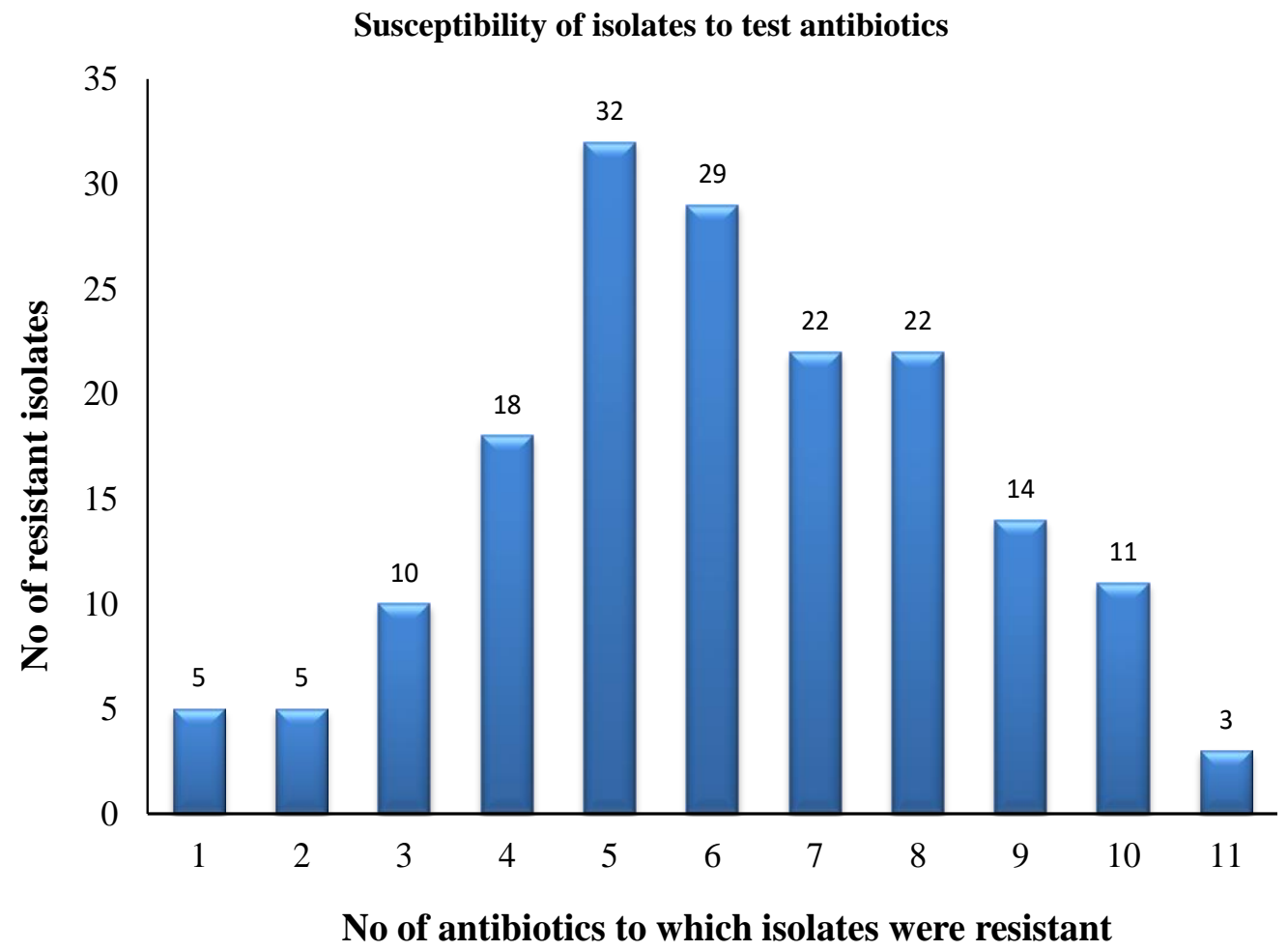

Figure 3: Multi-antibiotic resistant patterns of the staphylococcal isolates 
Table 1: Susceptibility of all the Staphylococcus isolates $(n=171)$ to given antibiotics by the disc diffusion test

\begin{tabular}{lcc}
\hline \multirow{2}{*}{ Test Antibiotic } & \multicolumn{2}{c}{ Susceptibility } \\
\cline { 2 - 3 } & No of Isolates & $\%$ \\
\hline Tetracycline $(10 \mu \mathrm{g})$ & 60 & 35.1 \\
\hline Erythromycin $(15 \mu \mathrm{g})$ & 89 & 52.0 \\
Cefoxitin $(30 \mu \mathrm{g})$ & 36 & 21.1 \\
Ciprofloxacin $(30 \mu \mathrm{g})$ & 151 & 88.3 \\
Ofloxacin $(5 \mu \mathrm{g})$ & 35 & 20.5 \\
Co-trimoxazole $(25 \mu \mathrm{g})$ & 108 & 63.2 \\
Vancomycin $(30 \mu \mathrm{g})$ & 125 & 73.1 \\
Gentamicin $(10 \mu \mathrm{g})$ & 112 & 65.5 \\
Oxacillin $(1 \mu \mathrm{g})$ & 67 & 39.2 \\
Penicillin $(1.5)$ & 14 & 8.2 \\
Cefotaxime $(30 \mu \mathrm{g})$ & 33 & 19.3 \\
\hline
\end{tabular}

Table 2: Multi-antibiotic Resistant Indices of test isolates ( $\mathrm{n}=171)$

\begin{tabular}{cccc}
\hline Number of antibiotics to which susceptible & MARI & No. of isolates & Cumulative \% at the MARI \\
\hline 1 & 0.09 & 5 & 2.92 \\
2 & 0.18 & 5 & 5.85 \\
3 & 0.27 & 10 & 11.70 \\
4 & 0.36 & 18 & 22.2 \\
5 & 0.45 & 32 & 40.94 \\
6 & 0.54 & 29 & 57.84 \\
7 & 0.64 & 22 & 70.76 \\
8 & 0.73 & 22 & 83.63 \\
9 & 0.81 & 14 & 92.81 \\
10 & 0.91 & 11 & 98.24 \\
11 & 1.00 & 3 & 100.0 \\
\hline
\end{tabular}

*Percentage of isolates with MARI > index value

However, subjecting them to Microgen ID test kit showed that some of the isolates identified as Staphylococcus aureus by the cultural method were non-Staphylococcus aureus staphylococcal species (non-SASS). A substantial proportion (62 out of 135) were inaccurately identified by the conventional method. While $S$. aureus and other Staphylococcus species were coagulase positive, the Microgen ID test kit was a more specific method of identifying $S$. aureus from other Staphylococcus species. The prevalence (33.6\% S. aureus and $32.7 \%$ MRSA) found in this study corroborates with the findings of Brown et al. [26] and Zanger et al. [27]. Prevalence study of microorganisms and in this case, $S$. aureus and specifically MRSA is undertaken to assess the level of the presence of MRSA in a population at a given time and determine the need for interventions. The carriers of MRSA are at risk of septicemia, wound infections and the occasional toxic shock syndrome [17]. MRSA infections have been linked to higher hospital costs, higher mortality rates and longer length of hospital stays [28-30]. Studies in different locations and different populations in Nigeria had MRSA prevalence either higher $[15,16,18]$ or lower $[14,17,19,20]$ than the prevalence observed in this study. However, those isolates were from clinical specimens or other anatomical sites

In Europe, prevalence of MRSA is either stabilizing or decreasing in most European countries. The population-weighted mean percentage was $16.4 \%$ in 2018, a decreasing trend from $19.0 \%$ in 2015 [31]. Some of European countries had MRSA prevalence of $\leq 10 \%$. The decreasing prevalence of MRSA over the years was achieved by most countries developing and implementing national recommendations and guidance documents on preventing the spread 
of MRSA, focusing on improving infection prevention and control practices and antibiotic stewardship.

For susceptibility test, ciprofloxacin demonstrated the greatest activity against all the isolates $(88.3 \%)$ while penicillin was least effective (Table 1). The activity of cotrimoxazole was comparable to those of ciprofloxacin and vancomycin. However, its performance was better than that of cefotaxime, a 3rd generation cephalosporin. This may be due to the limited prescription and use of co-trimoxazole especially with the advent of resistance. Co-trimoxazole may be a better alternative to vancomycin in the treatment of MRSA infections. However, Bishara et al [32] observed progressive increase in susceptibility of MRSA to cotrimoxazole from $31 \%$ in 1988 to $81 \%$ in 1997 in a retrospective study undertaken in Israel using blood samples. On the contrary, a study by Japoni et al [33] in Iran recorded low susceptibility of MRSA and Methicillin Susceptible $S$. aureus (MSSA) to cotrimoxazole, ciprofloxacin, tetracycline, erythromycin and gentamicin. The samples were obtained from multiple specimens such as blood, urine, wounds, nasal washing, abscess, and skin lesions. In Yenagoa, Nigeria, the study of $S$. aureus from urine samples indicated resistance of $80.4 \%$ to cotrimoxazole, $73.9 \%$ to gentamicin, $69.6 \%$ to vancomycin, $34.8 \%$ to ofloxacin and $32.6 \%$ to ciprofloxacin [34]. However, the abovementioned studies obtained samples from patients and not healthy volunteers. Generally, a high level of resistance is expected from hospital isolates. In addition, selective pressure of antibiotic use is higher in hospitals. A similar study undertaken in Ota, Nigeria with regards to participants who are healthy student volunteers indicated that $82.4 \%$ of MRSA isolates were resistant to co-trimoxazole and vancomycin [35]. However, the samples were swabs from the nose and neck. This is in contrast with our findings where $63.2 \%$ and
$73.1 \%$ of the urinary isolates were susceptible to co-trimoxazole and vancomycin, respectively (Table 1). Although both studies were undertaken with student community, the contrast may be due to variation in prior antibiotic exposure, pattern of prescriptions and policies of health facilities in the various locations. Oxacillin and preferably cefoxitin are used as markers for methicillin resistance. Therefore, $60.8 \%$ or $78.9 \%$ of the staphylococcal isolates were found to be Methicillin resistant using oxacillin or cefoxitin respectively (Table 1).

It was found that $94.15 \%$ of the isolates had MAR index $\geq 0.2$ (Table 2). This indicates that the isolates were probably from high-risk contamination source where antibiotics are heavily used, or the isolates have had previous exposure to antibiotics. The latter may be the case in the study population as people have easy access to antibiotics in Nigeria without prescriptions.

It was observed that out of 11 antibiotics utilized in this study, 6 of them had over $50 \%$ of the isolates resistant to them while only ciprofloxacin had less than $20 \%$ of the isolates resistant to it. Most of the isolates demonstrated resistance to at least 4 classes of antibiotics and consequently can be said to be not just multi-antibiotic resistant but also multi-drug resistant. This is an indicator of an emerging challenge in SA and MRSA related infections. Multidrug resistance is a threat to public health as selection of commercially available efficacious antibiotics for treatment of infections may be a herculean task. This finding corroborates the study of an epidemic clone of community-acquired methicillinresistant $S$. aureus USA300 published by Diep and co-workers [36]. USA300 was found to be resistant to $\beta$-lactams, fluoroquinolones, tetracycline, macrolide, clindamycin, and mupirocin; an ability facilitated by its acquisition of multiple resistance genes. In another study, Holden and co-workers [37] found in a variant of MRSA (TW20) a display 
of an extended antibiotic resistance pattern. TW20 displayed multidrug resistance to penicillin, methicillin, erythromycin, ciprofloxacin, gentamicin, neomycin, trimethoprim, and tetracycline.

World Health Organization had invited all countries to adopt strategies to prevent, control and monitor antimicrobial resistance [21]. Nigeria needs to set up a national body on antimicrobial resistance to collect, track and report antimicrobial resistance trends. It is in the interest of Nigeria that the effectiveness of available antibiotics is prolonged as bacteria develop resistance faster than new antibiotics are developed. In addition, the pharmaceutical companies have limited interest in developing antibiotics since microorganisms develop resistance to them in a short time and so antibiotic research and development is not a profitable venture. To contain antibiotic resistance, empirical treatment of infections must be reduced to only emergencies. Culture and susceptibility test should be undertaken, before prescriptions are given, with strict national quality assurance program for reporting laboratories. The regulatory authority should mitigate against poor quality antibiotics as sub-optimal concentrations of antimicrobial agent precipitates resistance. Antimicrobial resistance cannot be effectively contained without educating health professionals and patients. The strategies once proposed to combat irrational use of antibiotics should be enforced. The European surveillance report has shown that should Nigeria fully implement her National Action Plan on antimicrobial resistance, antimicrobial resistance can be contained.

Conclusion. A high percentage of healthy student volunteers from the University of Jos community are carriers of multi-drug resistant Staphylococcus aureus placing them at risk of severe infections. The isolates were better characterised to species level using the Microgen ID rapid test kit. MRSA isolates were multi-antibiotic resistant making selection of antibiotic for treatment of MRSA infections a challenge. For antimicrobial resistance to be contained, there must be a commitment from the healthcare professionals on antibiotic stewardship. Health policies should be made and implemented and such should include making antibiotics prescription only medicines which in turn will reduce the abuse of antibiotics. A national surveillance system is urgently needed to be up and running. Antimicrobial resistance is a major threat to public health that requires coordinated efforts of the government and the public.

\section{REFERENCES}

1. Grisold AJ, Leitner E, Mühlbauer G, Marth E, Kessler HH, "Detection of methicillin-resistant staphylococcus aureus and simultaneous confirmation by automated nucleic acid extraction and real-time PCR," Journal of clinical microbiology, vol. 40, no. 7, pp. 2392-2397, 2002.

2. Ibadin EE, Enabulele IO, Muinah F. "Prevalence of mecA gene among staphylococci from clinical samples of a tertiary hospital in Benin city, Nigeria," African health sciences, vol. 17, no. 4, pp. 1000-1010, 2017.

3. Tong SY, Davis JS, Eichenberger E, Holland TL, Fowler VG. "Staphylococcus aureus infections: Epidemiology, pathophysiology, clinical manifestations, and management," Clinical microbiology reviews, vol. 28, no. 3, pp. 603-661, 2015.

4. Taylor TA, Unakal CG. "Staphylococcus aureus," In: StatPearls [Internet]. Treasure Island (FL): StatPearls Publishing; 2019.

5. Ippolito G, Leone S, Lauria FN, Nicastri E, Wenzel RP. "Methicillin-resistant staphylococcus aureus: The superbug," International journal of infectious diseases, vol. 14pp. S7-S11, 2010.

6. Raygada JL, Levine DP. "Methicillin-resistant staphylococcus aureus: A growing risk in the hospital and in the community," American health \& drug benefits, vol. 2, no. 2, pp. 86, 2009.

7. Rasigade J, Vandenesch F. "Staphylococcus aureus: A pathogen with still unresolved issues," Infection, Genetics and Evolution, vol. 21pp. 510-514, 2014. 
8. Crossley KB, Jefferson KK, Archer GL, Fowler VG. Staphylococci in human disease. : John Wiley \& Sons, Singapore; 2009.

9. Lowy FD. "Staphylococcus aureus infections," New England journal of medicine, vol. 339, no. 8, pp. 520532, 1998.

10. Holt JG, Krieg NR, Sneath PH, Staley JT, Williams ST. "Bergey's manual of determinative bacteriology. 9th," Baltimor: William \& Wilkins, 1994.

11. Novick RP, Schlievert P, Ruzin A. "Pathogenicity and resistance islands of staphylococci," Microbes and Infection, vol. 3, no. 7, pp. 585-594, 2001.

12. Okeke IN, Lamikanra A, Edelman R. "Socioeconomic and behavioral factors leading to acquired bacterial resistance to antibiotics in developing countries," Emerging infectious diseases, vol. 5, no. 1, pp. 18, 1999.

13. Chokshi A, Sifri Z, Cennimo D, Horng H. "Global contributors to antibiotic resistance," Journal of global infectious diseases, vol. 11, no. 1, pp. 36, 2019.

14. Adetayo TO, Deji-Agboola AM, Popoola MY, Atoyebi TJ, Egberongbe KJ. "Prevalence of methicillin resistant staphylococcus aureus from clinical specimens in Ibadan, Nigeria," International Journal of Engineering Science, vol. 3 pp. 1-11, 2014.

15. Ajoke OI, Okeke IO, Odeyemi OA, Okwori A."Prevalence of methicillin-resistant staphylococcus aureus from healthy community individuals volunteers in jos south, Nigeria," Journal of Microbiology, Biotechnology and Food Sciences, vol. 9, no. 5, pp. 1389-1405, 2020.

16. Bunza NM, Isah AA, Hafsat MD, Asiya UI. "Antibiotic susceptibility pattern of staphylococcus aureus isolated from clinical samples in specialist hospital, sokoto," South Asian Journal of Research in Microbiology, pp. 1-6, 2019.

17. Ike B, Ugwu MC, Ikegbunam MN, Nwobodo D, Ejikeugwu C, Gugu T, Esimone CO. "Prevalence, antibiogram and molecular characterization of comunity-acquired methicillin-resistant staphylococcus aureus in Awka, Anambra Nigeria," The open microbiology journal, vol. 10pp. 211, 2016.

18. Osinupebi OA, Osiyemi JA, Deji-Agboola AM, Akinduti PA, Ejilude O, Makanjuola SO, Sunmola NO, and Osiyemi EO. "Prevalence of methicillin-resistant staphylococcus aureus in Abeokuta, Nigeria," South Asian Journal of Research in Microbiology, pp. 1-8, 2018.
19. Ghebremedhin B, Olugbosi MO, Raji AM, Layer F, Bakare RA, König B, König W. "Emergence of a community-associated methicillin-resistant staphylococcus aureus strain with a unique resistance profile in southwest Nigeria," Journal of clinical microbiology, vol. 47, no. 9, pp. 2975-2980, 2009.

20. Udeani TK, Onyebuchi CJ, Ikpenwa MC, Ezenwaka UR. "Prevalence and antibiotic susceptibility pattern of methicillin resistant staphylococcus aureus in burns and pressure ulcer patients," African Journal of Clinical and Experimental Microbiology, vol. 17, no. 2, pp. 130139, 2016.

21. World Health Organization, "Antimicrobial resistance global report on surveillance: 2014 summary," Antimicrobial resistance global report on surveillance: 2014 summary, 2014.

22. Chaudhary AS. "A review of global initiatives to fight antibiotic resistance and recent antibiotics' discovery," Acta Pharmaceutica Sinica B, vol. 6, no. 6, pp. 552-556, 2016.

23. Abubakar U, Tangiisuran B. "Nationwide survey of pharmacist's involvement in antimicrobial stewardship program in Nigerian tertiary hospitals," Journal of Global Antimicrobial Resistance, 2019.

24. Fadare JO, Ogunleye O, Iliyasu G, Adeoti A, Schellack N, Engler D, Massele A, Godman B. "Status of antimicrobial stewardship programmes in Nigerian tertiary healthcare facilities: Findings and implications," Journal of global antimicrobial resistance, vol. 17pp. 132-136, 2019.

25. CLSI. Performance Standards for Antimicrobial Susceptibility Testing; Twenty-Fifth Informational Supplement. CLSI document M100S25. Wayne, PA: Clinical and Laboratory Standards Institute; 2015.

26. Brown AF, Leech JM, Rogers TR, McLoughlin RM "Staphylococcus aureus colonization: Modulation of host immune response and impact on human vaccine design," Frontiers in immunology, vol. 4pp. 507, 2014.

27. Zanger P, Nurjadi D, Vath B, Kremsner PG. "Persistent nasal carriage of staphylococcus aureus is associated with deficient induction of human $\beta$ defensin 3 after sterile wounding of healthy skin in vivo," Infection and immunity, vol. 79, no. 7, pp. 2658-2662, 2011.

28. Thampi N, Showler A, Burry L, Bai AD, Steinberg M, Ricciuto DR, Bell CM, Morris AM. "Multicenter study of health care cost of patients admitted to hospital with staphylococcus aureus 
bacteremia: Impact of length of stay and intensity of care," American Journal of Infection Control, vol. 43, no. 7, pp. 739-744, 2015.

29. Rubio-Terres C, Garau J, Grau S, MartinezMartinez L., Cast of Resistance Study group, "Cost of bacteraemia caused by methicillin-resistant vs. methicillin-susceptible staphylococcus aureus in Spain: A retrospective cohort study," Clinical microbiology and infection, vol. 16, no. 6, pp. 722$728,2010$.

30. Nelson RE, Samore MH, Jones M, Greene T, Stevens VW, Liu C, Graves N, Evans MF, Rubin MA, "Reducing time-dependent bias in estimates of the attributable cost of health care-associated methicillinresistant staphylococcus aureus infections," Medical care, vol. 53, no. 9, pp. 827-834, 2015.

31. European Centre for Disease Prevention and Control. Surveillance of antimicrobial resistance in Europe 2018. 2019; https://www.ecdc.europa.eu/sites/default/files/docum ents/surveillance-antimicrobial-resistance-Europe2018.pdf Accessed 11th May, 2020.

32. Bishara J, Pitlik S, Samra Z, Levy I, Paul M, Leibovici L. "Co-trimoxazole-sensitive, methicillinresistant staphylococcus aureus, israel, 1988-1997," Emerging infectious diseases, vol. 9, no. 9, pp. 1168, 2003.

33. Japoni A, Ziyaeyan M, Jmalidoust M, Farshad S, Alborzi A, Rafaatpour N, Badiee P, "Antibacterial susceptibility patterns and cross-resistance of methicillin resistant and sensitive staphyloccus aureus isolated from the hospitalized patients in shiraz, iran," Brazilian Journal of Microbiology, vol. 41, no. 3, pp. 567-573, 2010.

34. Onanuga A, Awhowho GO. "Antimicrobial resistance of staphylococcus aureus strains from patients with urinary tract infections in Yenagoa, Nigeria," Journal of pharmacy \& bioallied sciences, vol. 4, no. 3, pp. 226, 2012.

35. Owolabi JB, Olorioke RC, "Prevalence and antimicrobial susceptibility of methicillin resistant staphylococcus aureus and coagulase-negative staphylococci isolated from apparently healthy university students in Ota, Nigeria," Prevalence, Vol. 5, No. 24, 2015.

36. Diep BA, Gill SR, Chang RF, Phan TH, Chen JH, Davidson MG, Lin F, Lin J, Carleton HA, Mongodin EF, "Complete genome sequence of USA300, an epidemic clone of community-acquired meticillin-resistant staphylococcus aureus," The Lancet, vol. 367, no. 9512, pp. 731-739, 2006.

37. Holden MT, Lindsay JA, Corton C, Quail MA, Cockfield JD, Pathak S, Batra R, Parkhill J, Bentley $\mathrm{SD}$, Edgeworth JD, "Genome sequence of a recently emerged, highly transmissible, multi-antibiotic-and antiseptic-resistant variant of methicillin-resistant staphylococcus aureus, sequence type 239 (TW)," Journal of Bacteriology, vol. 192, no. 3, pp. 888-892, 2010. 